\title{
Rama del canalis sinuosus imitando una reabsorcion intrarradicular: reporte de caso
}

\author{
Branch of the canalis sinuosus imitating an intrarradicular reabsorption: a case report \\ Daniela Ahumada-Tordecilla ${ }^{1, a}$.
}

\section{RESUMEN}

El objetivo de este estudio es potenciar la importancia del conocimiento anatómico clínico de las estructuras que conforman el maxilar anterior, particularmente el canalis sinuoso y sus ramas accesorias y como dichas estructuras se presentan en los exámenes imagenológicos complementarios bi y tridimensionales como la radiografía periapical y la Tomografía Computarizada de Haz Cónico (CBCT). Se presenta un caso clínico de superposición de una rama accesoria del canalis sinousus que simula una reabsorción radicular interna. El análisis minucioso de cada caso clínico, junto al uso de las herramientas imagenológicas actuales nos permite establecer diagnósticos clínicos certeros y evitar tratamientos innecesarios.

PALABRAS CLAVE: Canalis sinuoso, tomografía computarizada de haz cónico, reabsorción radicular.

\begin{abstract}
The objective of this study is to enhance the importance of clinical anatomical knowledge of the structures that conform the anterior maxilla, particularly the sinuous canalis and its accessory branches, and how these structures are presented in complementary two- and three-dimensional imaging examinations such as periapical radiography and Cone Beam Computed Tomography (CBCT). A clinical case of superposition of an accessory branch of the canalis sinousus that simulates internal root resorption is presented. The meticulous analysis of each clinical case, together with the use of current imaging tools, allows us to establish accurate clinical diagnoses and avoid unnecessary treatments.
\end{abstract}

KEYWORDS: Canalis sinuous, cone beam computed tomography, root reabsorption.

Facultad de Odontología, Universidad de Valparaíso. Valparaíso, Chile.

Cirujano Dentista, Licenciada en Odontología. 


\section{INTRODUCCIÓN}

El canalis sinuosus (CS) es un canal neurovascular, rama del canal infraorbitario a través del cual pasa el nervio alveolar anterosuperior, descrito por primera vez en 1939 por Wood-Jones (1). Este canalículo se origina en el canal infraorbitario posterior al foramen infraorbitario y discurre en dirección anterolateral, después de alcanzar la pared anterior del maxilar, el canal sinuoso se vuelve medial pasando por debajo del agujero infraorbitario cruzando la pared del antro de lateral a medial. Una vez que el conducto llega a la abertura nasal, se curva hacia abajo y desciende a lo largo de la abertura piriforme llegando al maxilar anterior (2,3). Aunque la presencia del canalis sinuosus es un aspecto anatómico normal, éste suele presentar canales accesorios (CA)(4), que discurren a través del hueso alveolar hacia el paladar anterior (figura 1), considerándose variantes anatómicas. Estudios previos enfocados en la presencia de canales adicionales reportan una prevalencia de un $15,7 \%$ a un $70,8 \%$ para la zona y aun así siguen siendo estructuras poco reconocidas por los odontólogos (5).

Tradicionalmente el maxilar anterior ha sido considerado un lugar relativamente seguro para ejecutar procedimientos quirúrgicos como cirugía de implantes, cirugía periodontal y endodóntica, extracción de supernumerarios, cirugía ortognática, entre otros. Sin embargo, la creciente tasa de intervenciones quirúrgicas en el área ha incrementado el reporte de lesiones iatrogénicas al canal sinuoso, principalmente neurovasculares (5), las cuales se asocian a hemorragias, parestesias, dolor postoperatorio, problemas de oseointegración e infección (6).

Los métodos de diagnóstico mediante imágenes convencionales como la radiografía periapical aunque proporcionan valiosa información sobre la región tienen ciertas limitaciones como superposiciones, distorsiones, ampliaciones, etc., las cuales pueden conducir a errores diagnósticos al simular por ejemplo, una lesión intra o perirradicular, repercutiendo directamente en el plan de tratamiento (7).

El desarrollo de las imágenes tridimensionales (3D) como la tomografía computarizada de haz (CBCT) y su mayor acceso en el ámbito odontológico, permiten una evaluación multiplanar detallada de alta resolución de la región maxilofacial, repercutiendo en el diagnóstico, planificación y tratamiento de los
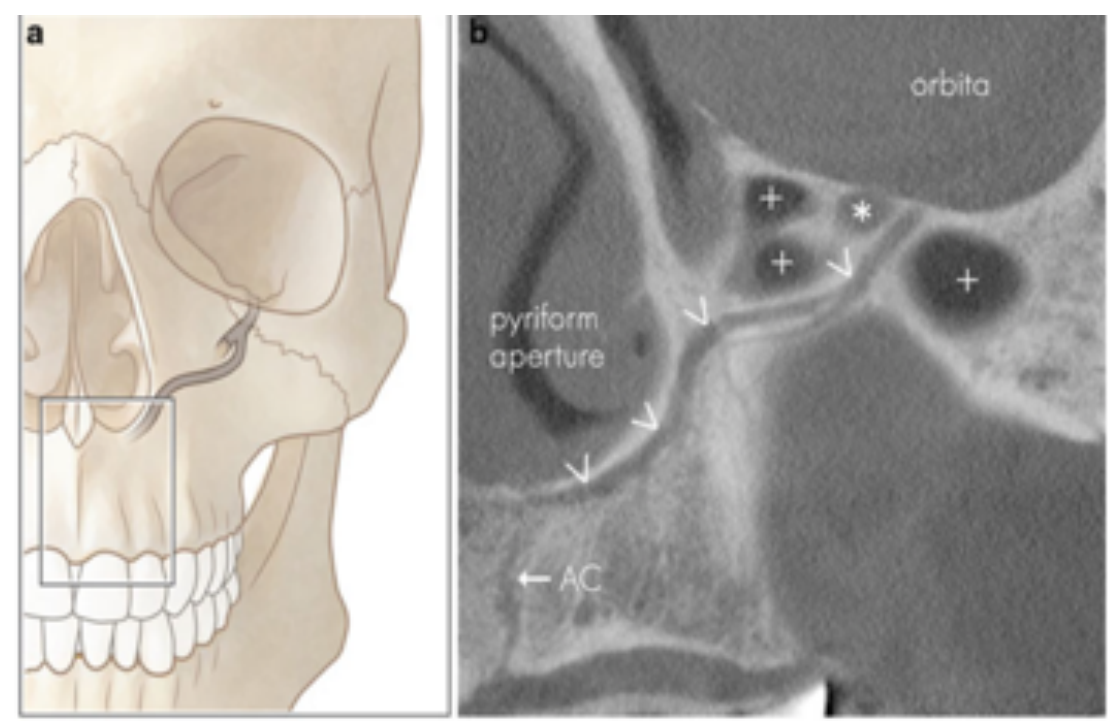

1a: Ilustración esquemática del canal sinuoso que se origina en el canal infraorbitario y se dirige hacia la apertura piriforme. 1b: CBCT: puntas de flecha: canal sinuoso, AC: Canal accesorio del CS, * conducto infraorbitario, +: bahías seno maxilar. (S. L. Thomas von Arx, «Anterior superior alveolar nerve (ASAN) A morphometric-anatomical analysis,» SWISS DENTAL JOURNAL, vol. SSO 125: 1202-1209, (2015).)

Figura 1. Ilustración esquemática del canal sinuoso 
pacientes. El CBCT permite mapear el CS y sus variantes, dando confianza al clínico con conocimiento anatómico de establecer diagnósticos certeros y planificar tratamientos quirúrgicos adecuados en caso de ser necesarios (8).

El objetivo de este reporte es recalcar la importancia del conocimiento clínico-anatómico de las estructuras que componen el macizo facial, particularmente en el paladar anterior y como se presenta en los exámenes imagenológicos bi y tridimensionales al evidenciar como la superposición radiográfica de la apertura palatina de una rama accesoria del canal sinuoso imita una reabsorción radicular interna en el diente 22 en una imagen convencional.

\section{REPORTE DE CASO}

Paciente masculino de 37 años, sin antecedentes mórbidos relevantes, acude a control odontológico de rutina en consulta privada para examen clínico y radiográfico, donde por sospecha de caries mesial se solicita radiografía periapical de diente 22 y bitewing bilateral de forma complementaria. Tras obtener las imágenes el informe radiológico describe: "P 22. Conducto permeable, a nivel de la unión del tercio apical y medio del conducto se observa pequeña área radiolúcida redondeada ¿canal accesorio, endorizálisis?, Espacio periodontal apical algo ensanchado. Se sugiere comparar con radiografías anteriores" (figura 2).

Como antecedente clínico el paciente relata haber estado bajo tratamiento ortodóncico fijo por 6 meses el año 2018 donde la radiografía panorámica solicitada para el estudio no presentaba alteración en la zona de interés. Con este informe el paciente es derivado con la endodoncista del centro, quien sospecha de una endorrizálisis e indica que el tratamiento estándar para dicho cuadro es el tratamiento de conducto.

El paciente ante lo expuesto busca una segunda opinión y donde tras solicitar una tomografía computarizada de haz cónico (CBCT) para confirmar el diagnóstico y realizar la derivación correspondiente, el informe radiológico del CBCT determinó que el diente 22 no presentaba un defecto reabsortivo endorradicular, ni conductos pulpares accesorios,

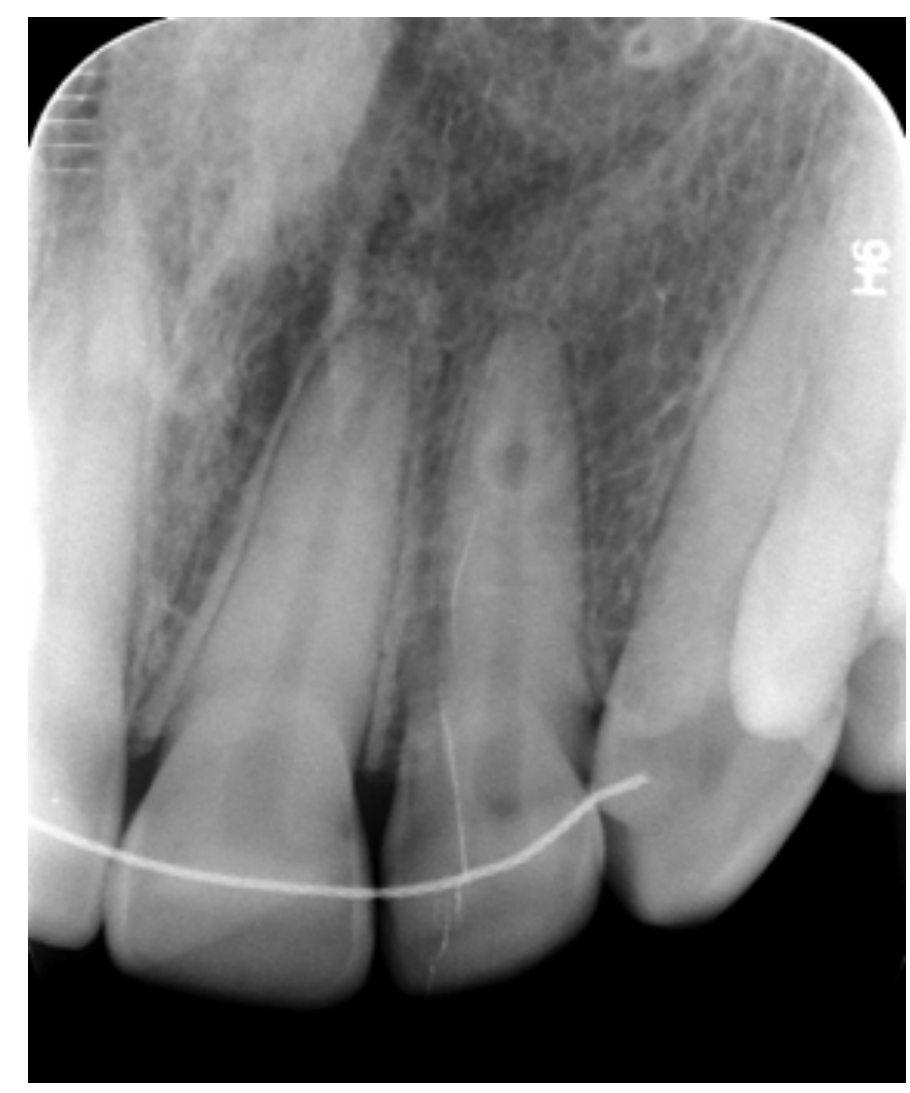

Figura 2. Radiografía periapical diente 22 con área radiolúcida redondeada en conducto radicular a nivel de tercio medio/apical. 
descartando de lleno el diagnóstico presuntivo y la necesidad del tratamiento endodóntico.

Al realizar la evaluación secuencial de los cortes multiplanares del CBCT (software Blue Sky Plan 4, versión 4.3.10) se aprecia un canal óseo corticalizado originado $1 \mathrm{~mm}$ bajo la base nasal del lado izquierdo en el hueso maxilar, $5 \mathrm{~mm}$ posterior a su borde anterior, de $1 \mathrm{~mm}$ de diámetro por $14 \mathrm{~mm}$ de largo aproximadamente, con dirección al paladar anterior y apertura palatina a nivel del tercio medio radicular de la pieza 22 (Figura 3), correspondiente a un canal accesorio del canalis sinuosus. Por tanto, se infirió que la superposición de esta estructura, con

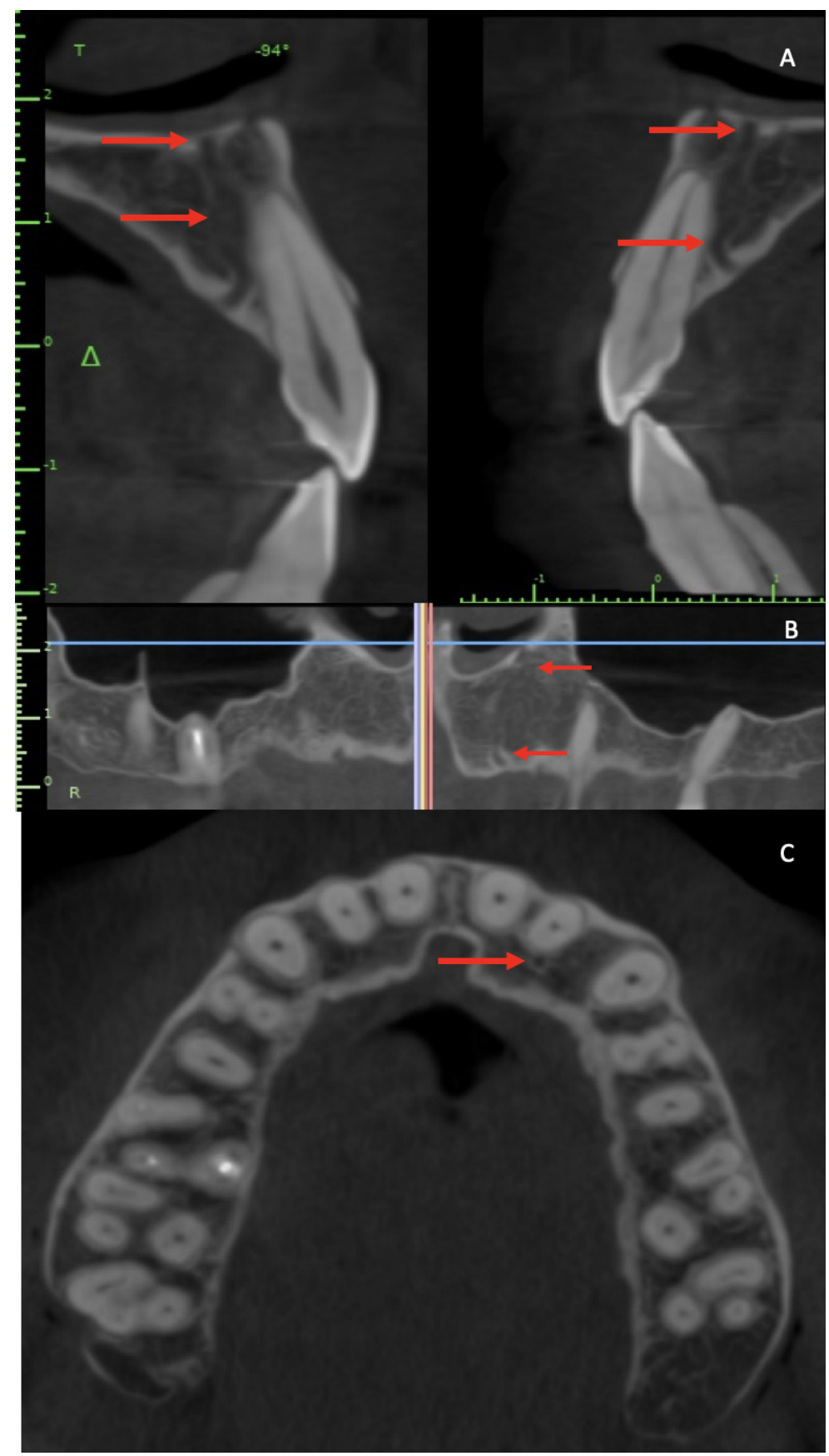

Figura 3. Cortes multiplanares tomografía computarizada. 3A: Cortes transaxiales, canal óseo accesorio palatino a diente 2.2, rama del canalis sinuosus. 3B: Reformación panorámica, canal accesorio desde base nasal a paladar anterior. 3C: Corte transversal, canal accesorio palatino a pieza 2.2 . 
la porción radicular de la pieza 22 más la angulación de la radiografía periapical imitó una endorrizalisis al examen radiográfico bidimensional.

Se informó al paciente el diagnóstico, el cual no requería de intervención terapéutica y se indicaron controles clínicos anuales de rutina.

\section{DISCUSION}

Aunque la presencia del canalis sinuosus es un aspecto anatómico normal, su apertura en el paladar anterior es una variación anatómica que no se ha descrito a cabalidad en la literatura. El mayor acceso a CBCT en el área odontológica hoy en día enfatiza la importancia que el clínico conozca e identifique correctamente dichas estructuras, considerando la alta prevalencia del CS y su alta frecuencia de canales accesorios en pro de establecer diagnósticos certeros y planes de tratamiento adecuados $(9,10,11,12,13)$.

La tomografía computarizada de haz cónico proporciona como examen complementario una visualización precisa y detallada de las estructuras maxilomandibulares permitiendo al odontólogo realizar un diagnóstico y plan de tratamiento ideal para cada caso clínico, previniendo principalmente posibles complicaciones a nivel quirúrgico (5).

La evaluación radiográfica tridimensional e identificación preoperatoria es esencial en la planificación de procedimientos quirúrgicos que involucran la zona maxilar anterior al disminuir el riesgo de complicaciones como hemorragia intraoperatoria, cambios en la sensibilidad global del territorio del Nervio Alveolar Anterior Superior, dificultad en la osteointegración de los implantes (14), entre otras. Sumado a lo anterior, reportes existentes sobre las variaciones anatómicas del CS indican que éstas pueden simular lesiones intra o perirradiculares por superposición en imágenes bidimensionales y conducir a tratamientos innecesarios (15).

Actualmente existen 33 publicaciones en la base de datos Pubmed relacionadas con el canalis sinuosus, donde el $60 \%$ de ellos fueron publicados en los últimos 5 años. De los resultados obtenidos, 20 de ellos se asocian al uso de CBCT como herramienta diagnóstica evidenciando el mayor acceso a exámenes imagenológicos tridimensionales y el incremento de intervenciones quirúrgicas ligadas al ámbito de la cirugía e implantología. Según lo informado por estos estudios se desprende que la prevalencia de canales accesorios del canalis sinuosus varía desde un 15,7\% a un $70,8 \%$, los tamaños de muestra fluctúan desde reportes de casos a análisis de hasta $1460 \mathrm{CBCT}$ (8); y el parámetro diámetro considerado en todos los estudios va desde 0.5 a $1 \mathrm{~mm}$.

En relación al análisis estadístico, el criterio de género presenta resultados estadísticamente significativos en los estudios de Aoki et al., (2020), Shah et al., (2020), Tomrukçu et al., (2020), Gurler et al., (2017), Von Arx et al., (2013) y Machado et al., (2016) en cuanto a predominio del género masculino sobre el femenino en relación a frecuencia de CA $(9,15,16,17,18,19)$, a diferencia de los estudios de Oliveira-Santos et al., (2013) y Orhan et al., (2018) quienes exponen no encontrar diferencias entre géneros $(2,7)$. El parámetro de edad en la mayoría de los estudios no es estadísticamente significativo $(2,8,9,16,19)$, sin embargo, Von Arx et al., informa una mayor frecuencia del CS y sus ramas accesorias en individuos adultos en comparación con menores de edad, aunque su tamaño muestral reducido podría estar sujeto a valoración. Este reporte coincide con lo expuesto anteriormente en relación a prevalencia de género y diámetro obtenido el cual cumple con el valor promedio general de $1 \mathrm{~mm}$.

En síntesis, el conocimiento de las estructuras anatómicas y sus variantes junto al uso de las herramientas imagenológicas disponibles actualmente nos permiten mediante el análisis minucioso de cada caso clínico, establecer diagnósticos certeros y planes de tratamiento adecuados, disminuyendo el riesgo de complicaciones, tratamientos innecesarios y permitiendo la correcta planificación de cada intervención en virtud del bienestar de cada paciente $(10,18)$.

Conflicto de intereses: La autora declara no tener ningún conflicto de interés

Aprobación ética: El paciente proporcionó su consentimiento informado por escrito.

Financiamiento: Ninguno

Contribuciones de los autores: Autor único

Correspondencia:

Dra. Daniela Ahumada Tordecilla 
Lord Cochrane 30, oficina 203, Santiago, Chile. Correo electrónico: Daniela.ahumada.tordecilla@ gmail.com

\section{REFERENCIAS BIBLIOGRÁFICAS}

1. Jones FW. The anterior superior alveolar nerve and vessels. J Anat. 1939;73(Pt 4):583-91.

2. de Oliveira-Santos C, Rubira-Bullen IR, Monteiro SA, León JE, Jacobs R. Neurovascular anatomical variations in the anterior palate observed on CBCT images. Clin Oral Implants Res. 2013;24(9):1044-8.

3. Von Arx T, Lozanoff S. Anterior superior alveolar nerve (ASAN). Swiss Dent J. 2015;125(11):1202-9.

4. Rusu MC, Iacov-Crăiţoiu MM, Săndulescu $M$, Cârstocea L, Stana DM. Constant features of the adult maxillary bone in the site of the premaxillary suture: the sutura notha, Macalister's foramina, Parinaud's canal, and the second angle of the canalis sinuosus of Wood Jones. Rom J MorpholEmbryol. 2019;60(4):10971103.

5. Shan $\mathrm{T}, \mathrm{Qu}$ Y, Huang $\mathrm{X}, \mathrm{Gu}$ L. Cone beam computed tomography analysis of accessory canals of the canalis sinuosus: A prevalent but often overlooked anatomical variation in the anterior maxilla. J Prosthet Dent. 2020;1:S0022-3913(20)30435-2. doi: 10.1016/j. prosdent.2020.05.028

6. Lopes Dos Santos G, Ikuta CRS, Salzedas LMP, Miyahara GI, Tjioe KC. Canalis sinuosus: An Anatomic Repair that May Prevent Success of Dental Implants in Anterior Maxilla. J Prosthodont. 2020; 29(9):751-755. doi: 10.1111/jopr.13256

7. Orhan K, Gorurgoz C, Akyol M, Ozarslanturk S, Avsever H. An anatomical variant: evaluation of accessory canals of the canalis sinuosus using cone beam computed tomography. Folia Morphol (Warsz). 2018;77(3):551-557. doi: 10.5603/FM.a2018.0003

8. Wanzeler AM, Marinho CG, Alves Junior SM, Manzi FR, Tuji FM. Anatomical study of the canalis sinuosus in 100 cone beam computed tomography examinations. Oral Maxillofac Surg. 2015; 19(1):49-53. doi: 10.1007/ s10006-014-0450-9

9. Aoki R, Massuda M, Zenni LTV, Fernandes KS. Canalis sinuosus: anatomical variation or structure? Surg Radiol Anat. 2020;42(1):69-74.

10. Baena-Caldas G P, Rengifo-Miranda H,L, HerreraRubio AM, Peckham X, Zúñiga J R. Frecuencia de Canalis Sinuosus y sus variaciones anatómicas en imágenes de tomografía computarizada de haz cónico. Int J Morphol. 2019; 37(3):852-857.
11. Ferlin R, Pagin BSC, Yaedú RYF. Canalis sinuosus: a systematic review of the literature. Oral Surg Oral Med Oral Pathol Oral Radiol. 2019;127(6):545-551.

12. Ghandourah AO, Rashad A, Heiland M, Hamzi BM, Friedrich RE. Cone-beam tomographic analysis of canalis sinuosus accessory intraosseous canals in the maxilla. Ger Med Sci. 2017;15: 20.

13. Machado VC, Chrcanovic BR, Felippe MB, Manhães Júnior LR, de Carvalho PS. Assessment of accessory canals of the canalis sinuosus: a study of 1000 cone beam computed tomography examinations. Int J Oral Maxillofac Surg. 2016;45(12):1586-1591.

14. Olenczak JB, Hui-Chou HG, Aguila DJ 3rd, Shaeffer CA, Dellon AL, Manson PN. Posttraumatic Midface Pain: Clinical Significance of the Anterior Superior Alveolar Nerve and Canalis Sinuosus. Ann Plast Surg. 2015;75(5):543-7.

15. Shah PN, Arora AV, Kapoor SV. Accessory branch of canalis sinuosus mimicking external root resorption: A diagnostic dilemma. J Conserv Dent. 2017;20(6):479481 .

16. Tomrukçu DN, Köse TE. Assesment of accessory branches of canalis sinuosus on CBCT images. Med Oral Patol Oral Cir Bucal. 2020;25(1):e124-e130. doi: 10.4317/medoral.23235

17. Gurler G, Delilbasi C, Ogut EE, Aydin K, Sakul U. Evaluation of the morphology of the canalis sinuosus using cone-beam computed tomography in patients with maxillary impacted canines. Imaging Sci Dent. 2017;47(2):69-74. doi: 10.5624/isd.2017.47.2.69. PMC5489671.

18. Von Arx T, Lozanoff S, Sendi P, Bornstein MM. Assessment of bone channels other than the nasopalatine canal in the anterior maxilla using limited cone beam computed tomography. Surg Radiol Anat. 2013;35(9):783-90.

19. Machado VC, Chrcanovic BR, Felippe MB, Manhães Júnior LR, de Carvalho PS. Assessment of accessory canals of the canalis sinuosus: a study of 1000 cone beam computed tomography examinations. Int J Oral Maxillofac Surg. 2016;45(12):1586-1591.

Recibido : 15-06-2021

Aceptado : 28-08-2021 